Chapter 15

\title{
Photonics of Heterogeneous Dielectric Nanostructures
}

\author{
Vladimir Dzyuba, Yurii Kulchin and \\ Valentin Milichko \\ Additional information is available at the end of the chapter \\ http://dx.doi.org/10.5772/50212
}

\section{Introduction}

Over last 20 years great scientific attention has been paid to nanostructures and nanocomposites based on nanoparticles of semiconductor materials $\left(1 \mathrm{eV}<\mathrm{E}_{\text {gap }}<3 \mathrm{eV}\right)$ since they exhibit a wide range of nonlinear properties and can be used in various applied fields [1-5]. However, the history of active investigation of dielectric nanostructures' properties started only recently [6-8]. These structures are the heterogeneous medium formed by liquid or solid dielectric matrices (e.g., polymer glasses and oils) and nanoparticles of dielectrics $\left(\mathrm{Al}_{2} \mathrm{O}_{3}, \mathrm{SiO}_{2}, \mathrm{MgO}\right.$, etc.).

As some experiments have shown $[6,8,9]$, such structures have nonlinear optical properties, whose dependence on the intensity and optical radiation wavelength is not typical of previously known nonlinear optical media [10-14]. The anomalous nonlinear optical properties are manifested in the fact that, firstly, the nanostructures' optical response on the radiation is of a non-thermal nature and occurs at radiation intensities below $1 \mathrm{~kW} / \mathrm{cm}^{2}$ [9]. Second, despite the wide band gap of nanostructures' components $\left(\mathrm{E}_{\mathrm{gap}}>3 \mathrm{eV}\right)$, this response takes place in the visible and infrared region of light spectrum, and reaches a maximum then decreases to zero under increasing intensity $[8,9,15-17]$.

The dielectric nanostructures show other unexpected nonlinear optical properties. The nonlinear interaction of high-intensity radiation of different frequencies results in the generation of harmonics in conventional dielectric media. In the case of propagation of the low-intensity radiation of different frequencies in the dielectric nanostructures, the nonlinear interaction is manifested in the dependence of the light beam intensity on the intensity of another collinearly propagating light beam [18]. The two-frequency interaction observed in nano- 
structures does not prevent the generation of harmonics, but this process requires radiation intensities four orders of magnitude higher.

The optical nonlinearity of dielectric nanostructures allows one to believe that they will be used to develop and create new optoelectronic [19-24] and fibre-optic devices to control [11], process and transmit the information [25]. Of no less interest are the prospects of using such nanostructures in new optical materials with controlled optical properties, in particular, photonic crystals [26] and the media generating optical solitons at low intensities. In addition, several international research groups have proposed using these structures to create the elements of electrical circuits, since the nonlinear properties appear in the range of $\mathrm{THz}$ and $\mathrm{GHz}$ radiation and under the influence of an electric potential [27-30].

The study of nonlinear optical properties of dielectric nanostructures containing nanoscale objects of different chemical natures, shapes and sizeshas shown that the existence of a lowthreshold optical response is due to a number of conditions. The first is the presence of defect levels in the band gap of nanoparticles' charge carriers and this is manifested in the form of absorption bands in the nanoparticles' transmission spectrum [8,9,31-33]. Second, the radiation forming the nonlinear response of the nanostructure must have a frequency lying within the absorption band [8,9]. Third, the size and shape of the nanoparticles have to lead to the formation of a wide range of exciton states due to the quantum size effect [10,34-38]. Fourth, the matrix permittivity must be less than that of nanoparticle material, since the chemical nature of the matrix material significantly affects the formation of longlived exciton states [9,31,39-42]. Fifth, the value of electric dipole moments induced by electrons phototransition should be substantially larger than dipole moments in the bulk material. It allows observing the optical nonlinearity of nanostructures with a low concentration of nanoparticles under low-intensity optical fields.

The theoretical description of the observed effects [43-45] is based on the fact that the occurrence of nontypical optical nonlinearity requires the existence of defect levels and the broad band of exciton states in the energy band gap of charge carriers. The radiation causes the electron transitions from the defect to the exciton levels, thereby creating the photo-induced population difference. This process is accompanied by the appearance of the nanoparticle electric dipole moment, herewith its module depends nonlinearly on the intensity and light wavelength. The theory conclusions and theoretical modelling of transmission spectrum and the behaviour of the nonlinear refractive index are very similar to the experimental results [9].

It follows from the theory that the nature of the nonlinearity is determined not only by the behaviour of the photo-induced dipole moment module in an external field, but also by the nanoparticle's orientation along the vector E. However, this orientation has a minor contribution to the nonlinearity, so the observed nonlinear optical response can take place in the case of unpolarized light and solid nanostructures, which is in agreement with the experiment.

This chapter is an original quantitative study of the nonlinear refraction and absorption of continuous low-intensity laser radiation in different heterogeneous dielectric nanostructures and compares these data with theoretical ones. In addition, the theory of nonlinear light transmission by dielectric nanostructures is discussed. 


\section{Experimental Chapter}

\subsection{Dielectric Nanocomposite Preparation and Spectral Features}

To study the changes of optical characteristics of the heterogeneous dielectric nanostructuresunder continuous low-intensity radiation we used the dielectric $\mathrm{Al}_{2} \mathrm{O}_{3}, \mathrm{SiO}_{2}, \mathrm{TiO}_{2}$ and $\mathrm{ZnO}$ nanoparticles with 7,$2 ; 8 ; 3,4$ and $3,3 \mathrm{eV}$ band gaps of the bulk samples respectively [46]. The nanoparticles were purchased from Sigma Aldrich Company and investigated by AFM microscopy (Figures 2,3). The $\mathrm{Al}_{2} \mathrm{O}_{3}$ nanoparticles were used from work [9]. The averaged dimensions of nanoparticles are $45 \mathrm{~nm}$ in diameter and $6 \mathrm{~nm}$ in height for $\mathrm{Al}_{2} \mathrm{O}_{3}, 20 \mathrm{~nm}$ in diameter and $10 \mathrm{~nm}$ in height for $\mathrm{SiO}_{2}, 15 \mathrm{~nm}$ in diameter and $5 \mathrm{~nm}$ in height for $\mathrm{TiO}_{2}$ and $50 \mathrm{~nm}$ in diameter and $40 \mathrm{~nm}$ in height for $\mathrm{ZnO}$. As a matrix for nanoparticles, dielectric immersion and transformer oils were used. The immersion oil consists of weak polar molecules and is based on cedar resin witha negative temperature gradient of refractive index $|\mathrm{dn} / \mathrm{dT}|=4^{*} 10^{-4}$. The transformer oil is a PDMS (polydimethylsiloxane) liquid having an optical transparency up to $200 \mathrm{~nm}$, chemical inertness, a high heat resistance $\left(|\mathrm{dn} / \mathrm{dT}|<10^{-7}\right)$ and high stability of dielectric characteristics.

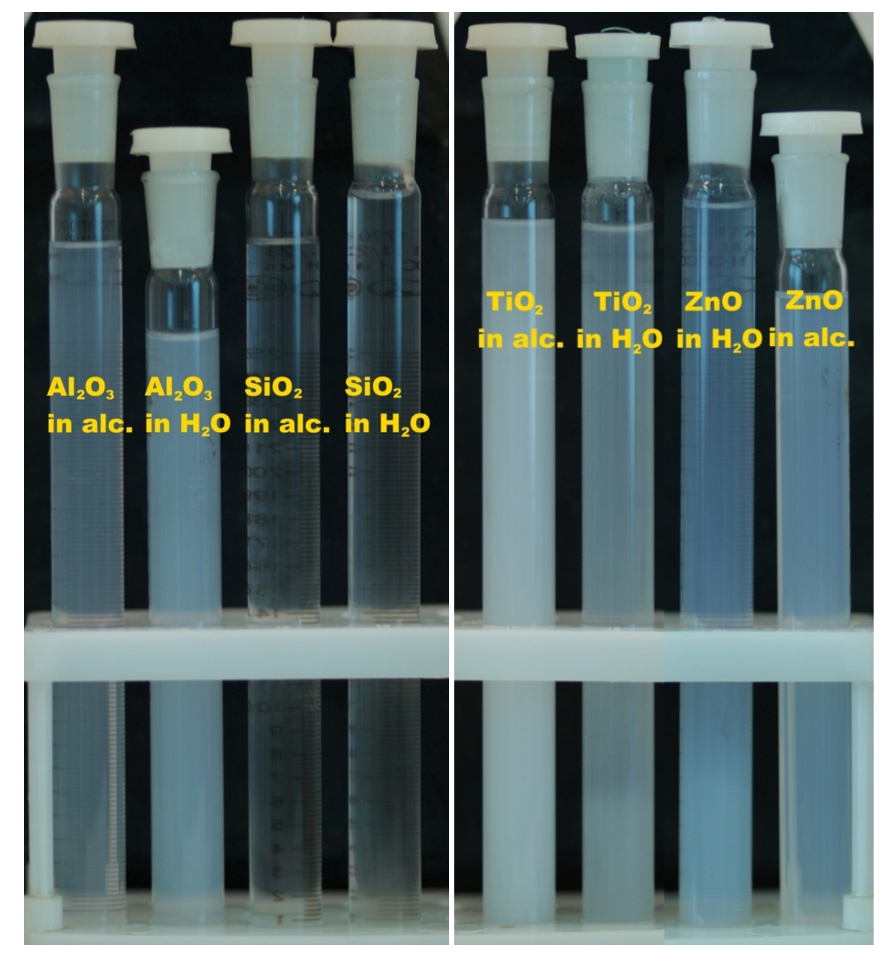

Figure 1. The nanoparticles suspension in isopropyl alcohol and distilled water. 
The nanopowders were dissolved in isopropyl alcohol to precipitate the particles sticking together (Figure 1). After precipitation the upper isopropyl alcohol layers containing separate nanoparticles with small deviation in size were added into the oil $\left(\mathrm{Al}_{2} \mathrm{O}_{3}\right.$ into immersion oil; $\mathrm{SiO}_{2}, \mathrm{TiO}_{2}$ and $\mathrm{ZnO}$ into PDMS) pre-heated to $40^{\circ} \mathrm{C}$. Slow heating of the mixture to $60^{\circ} \mathrm{C}$ resulted in the appearance of convection currents which, in turn, form the uniform nanoparticles' distribution over the entire suspension volume and lead to alcohol evaporation. Then heterogeneous dielectric nanostructures (hence forth called the HDN) with nanoparticle volume concentration $>1 \%$ were placed in a quartz cuvette $5 \mathrm{~mm}$ thick and $18.7 \mathrm{~mm}$ in length.

The nonlinear response of the medium on the radiation of certain frequencies can take place if this medium has nonlinear spectral characteristics that are related directly to the energy spectrum structure of charge carriers. When dealing with a nanoparticle we can expect the energy spectrum of its charge carriers to depend on the form and degree of nanoparticle surface development. Besides, the energy spectrum will depend on the matrix material and, to a greater extent, on its permittivity $\varepsilon$. Therefore, we studied the transmission spectra of $\mathrm{Al}_{2} \mathrm{O}_{3}$ (permittivity of the bulk sample is $\left.\varepsilon_{\text {stat }}=10\right), \mathrm{SiO}_{2}\left(\varepsilon_{\text {stat }}=4,5\right), \mathrm{TiO}_{2}\left(\varepsilon_{\text {stat }}>86\right)$ and $\mathrm{ZnO}$ $\left(\varepsilon_{\text {stat }}=8,8\right)$ nanoparticles suspended in isopropyl alcohol $\left(\varepsilon_{\text {stat }}=24\right)$, distilled water $\left(\varepsilon_{\text {stat }}=80\right)$ and oil $\left(\varepsilon_{\text {stat }}=2,5\right)$.

As it follows from the transmission spectra figures (Figure 4), the nanoparticles of broadband dielectrics $\left(\mathrm{Al}_{2} \mathrm{O}_{3}\right.$ and $\left.\mathrm{SiO}_{2}\right)$ suspended in oil have a non-symmetric broad absorption band that is formed by exciton states with high density. The asymmetry of the absorption band is explained due to the broadening of the exciton levels. This band is not observable either in the bulk sample or in the nanoparticles' array suspended in other media.This can be explained by the fact that the electronic structure of nanoparticles embedded in a matrix depends strongly on the ratio between the permittivity of matrix $\varepsilon_{1}$ and nanoparticles $\varepsilon_{2}$ $[39,40]$. Given $\varepsilon_{1} / \varepsilon_{2}>1$, polarization interaction leads to attraction of positive charges to the inner surface of the nanoparticles and to the destruction of defect states by virtue of interaction the nanoparticle electrons from these levels with high-polarized matrix molecules. If $\varepsilon_{1} / \varepsilon_{2}<1$, polarization interaction causes repulsion of charges from the nanoparticle's surface into the interior, thus preserving these states. So, the propagation of visible radiation $(2,1 \mathrm{eV}<\mathrm{E}<3,1 \mathrm{eV})$ into the $\mathrm{HDN}$ based on oil and $\mathrm{Al}_{2} \mathrm{O}_{3}$ nanoparticles results in electron transition from defect to exciton levels (Figure 5). In the case of water and alcohol matrixes there are no free electrons on the defect level, therefore, electron transition under ultraviolet radiation $(\mathrm{E}>4 \mathrm{eV})$ is available. The same situation is exists for the $\mathrm{HDN}$ based on oil and $\mathrm{SiO}_{2}$ nanoparticles: the exciton generation occurs under visible and ultraviolet radiation $(2 \mathrm{eV}<\mathrm{E}<6 \mathrm{eV})$, however, less probable electron transitions from valence band to exciton levels can take place provided $\mathrm{E}>2 \mathrm{eV}$ (Figure 5).

The $\mathrm{TiO}_{2}$ and $\mathrm{ZnO}$ nanoparticles' array transmission spectra (Figure 6) inform that the nanoparticles of narrow-band dielectrics suspended in oil have a blurred edge of fundamental absorption that is formed by exciton states without any absorption band within (400-700)nm. The matrix permittivity effects only the position of fundamental absorption. 

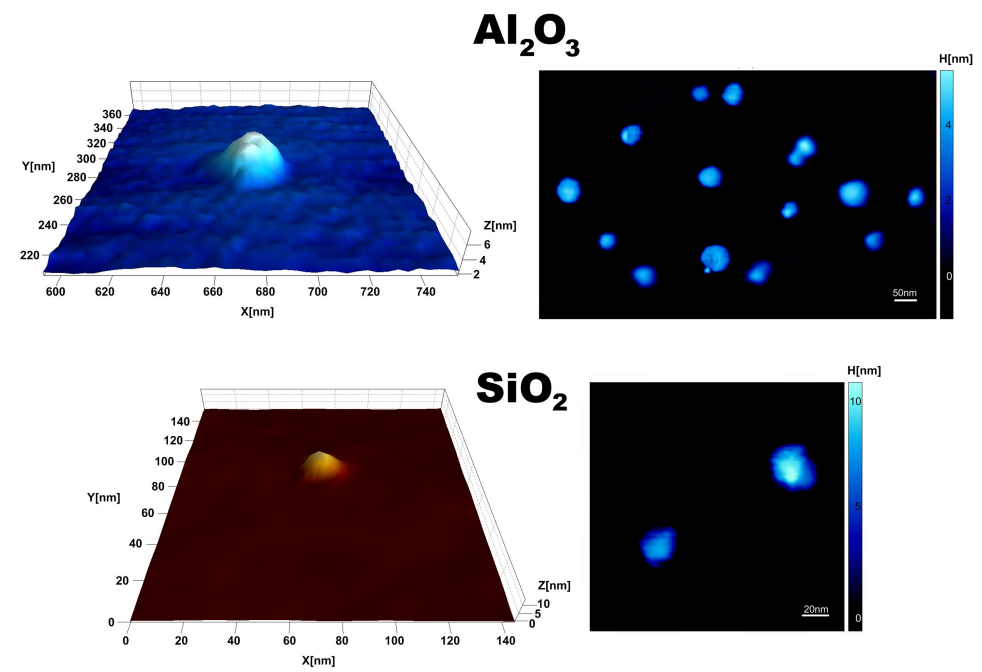

Figure 2. AFM images of $\mathrm{Al}_{2} \mathrm{O}_{3}$ and $\mathrm{SiO}_{2}$ nanoparticles precipitated on a mica place. Defined dimensions are $45 \mathrm{~nm}$ in diameter and $6 \mathrm{~nm}$ in height for $\mathrm{Al}_{2} \mathrm{O}_{3}, 20 \mathrm{~nm}$ in diameter and $10 \mathrm{~nm}$ in height for $\mathrm{SiO}_{2}$.
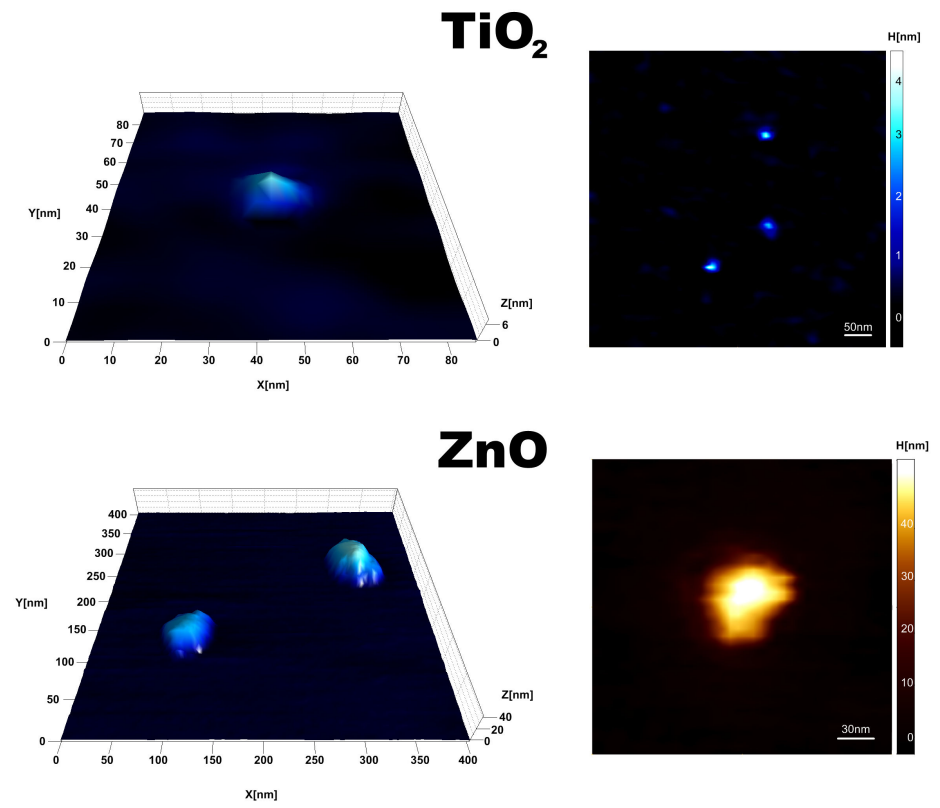

Figure 3. $\mathrm{AFM}$ images of $\mathrm{TiO}_{2}$ and $\mathrm{ZnOnanoparticles} \mathrm{precipitated} \mathrm{on} \mathrm{a} \mathrm{mica} \mathrm{place.} \mathrm{Defined} \mathrm{dimensions} \mathrm{are} 15 \mathrm{~nm}$ in diameter and $5 \mathrm{~nm}$ in height for $\mathrm{TiO}_{2}, 50 \mathrm{~nm}$ in diameter and $40 \mathrm{~nm}$ in height for $\mathrm{ZnO}$. 
$\mathbf{A}$

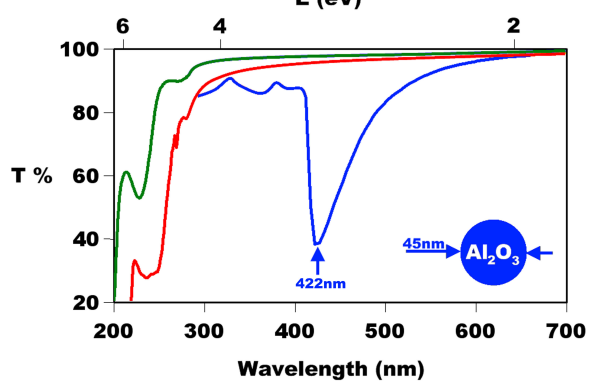

B

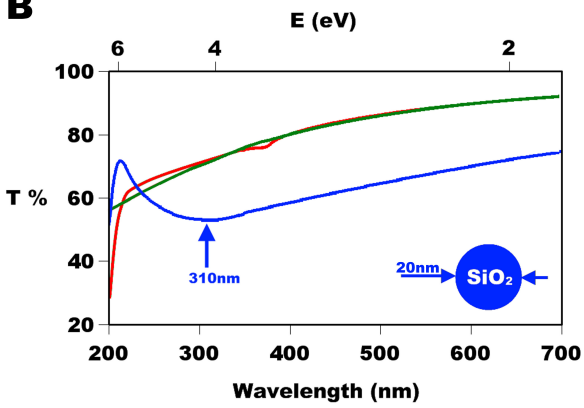

Figure 4. Transmission spectra of $\mathrm{Al}_{2} \mathrm{O}_{3}(\mathrm{~A})$ and $\mathrm{SiO}_{2}(\mathrm{~B})$ nanoparticles' array dispersed in $\mathrm{H}_{2} \mathrm{O}$ (green curve), isopropyl alcohol (red curve) and oil (blue curve). The curves were obtained by division of the T spectrum of nanoparticle suspension by that of the matrix.
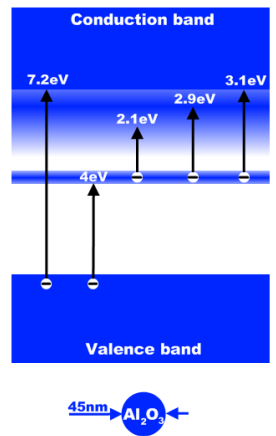

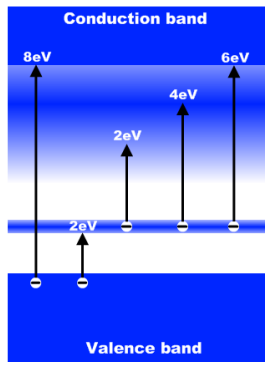

$\stackrel{20 \mathrm{~nm}}{\longrightarrow \mathrm{SiO}_{2}} \leftarrow$

Figure 5. The energy band gap structure of $\mathrm{Al}_{2} \mathrm{O}_{3}$ and $\mathrm{SiO}_{2}$ nanoparticles' array.

A

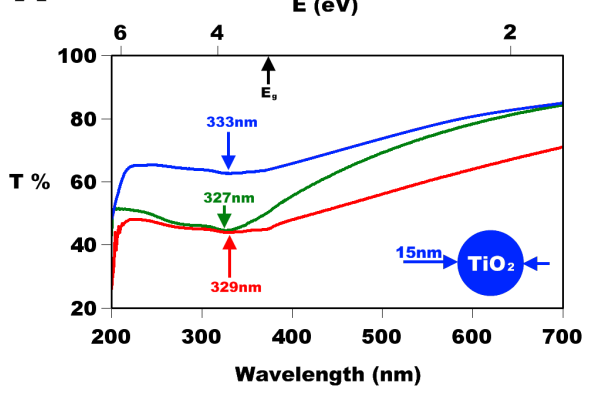

B

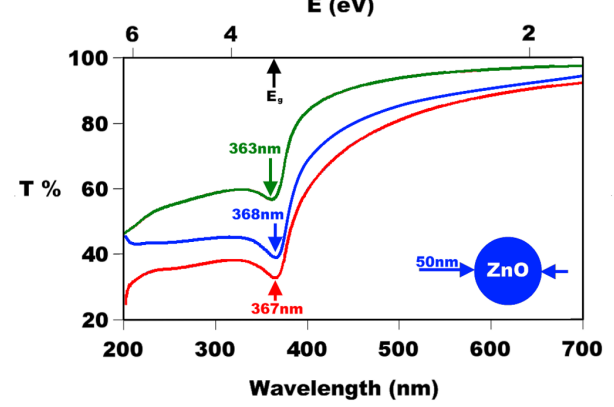

Figure 6. Transmission spectra of $\mathrm{TiO}_{2}(\mathrm{~A})$ and $\mathrm{ZnO}(\mathrm{B})$ nanoparticles' array dispersed in $\mathrm{H}_{2} \mathrm{O}$ (green curve), isopropyl alcohol (red curve) and oil (blue curve). The curves were obtained by division of the T spectrum of nanoparticle suspension by that of the matrix. 


\subsection{Z-scan Experiment}

The experimental study of the dependence of the HDN nonlinear optical response on the laser radiation of variable intensity was performed using the standard z-scan technique with open and closed apertures [47,48] (Figure 7). Since the nonlinear optical response of the HDN appears under low-intensity optical fields we used the semiconductor lasers providing focal intensities of up to $500 \mathrm{~W} / \mathrm{cm}^{2}$ for green and violet radiation. Such low intensities are at least four orders of magnitude lower than the pulsed mode intensity required for appearance of the nonlinear response in previously known environments [10-14].

In the z-scan experiments, the $\mathrm{HDN}$ based on $\mathrm{Al}_{2} \mathrm{O}_{3}, \mathrm{SiO}_{2}, \mathrm{TiO}_{2}$ and $\mathrm{ZnO}$ nanoparticles can be considered as thin experimental samples, since the interaction length of radiation with the samples is equal to $L=5 \mathrm{~mm}$, being less than minimum Rayleigh range [47] $Z_{0}=\left(\pi \omega_{0}^{2}\right) /$ $\lambda=8.7 \mathrm{~mm}$.

The values the changes in refractive index $\Delta \mathrm{n}(\mathrm{I}, \lambda)$ and absorption coefficient $\Delta \alpha(\mathrm{I}, \lambda)$ one can calculate by the following:

$$
\begin{gathered}
\Delta n(I, \lambda)=\frac{\lambda \Delta T_{p v} \ln ^{I_{0}} /}{0.812 \pi(1-S)^{0.27} L\left(1-{ }^{I} / I_{0}\right)} \\
\Delta a(I, \lambda)=\frac{2 \sqrt{2} \Delta T}{L}
\end{gathered}
$$

that has been derived from the number of expressions:

$$
\begin{gathered}
\Delta T_{p v} \approx 0.406(1-S)^{0.27}\left|\Delta \Phi_{0}\right| \\
\Delta \Phi_{0}=\frac{2 \pi}{\lambda} n_{2} I_{0} L_{\text {eff }}
\end{gathered}
$$

where $\lambda$ is a radiation wavelength and $\mathrm{I}_{0}$ and Iare the input and output intensities, respectively. $\mathrm{S}$ is a fraction radiation transmitted by the aperture in the absence of the sample ( $\mathrm{S}=0.04$ and 0.06 provided the $\mathrm{HDN}$ based on $\mathrm{Al}_{2} \mathrm{O}_{3}$ in green and violet optical field, respectively; $\mathrm{S}=0.22$ and 0.35 provided the $\mathrm{HDN}$ based on $\mathrm{SiO}_{2}, \mathrm{TiO}_{2}, \mathrm{ZnO}$ in green and violet optical field, respectively), $\mathrm{L}_{\text {eff }}=\mathrm{L}^{*}\left(1-\mathrm{e}^{-\alpha \mathrm{L}}\right), \mathrm{L}$ and $\alpha$ are the sample length and absorption coefficient, respectively and $\Delta \mathrm{T}$ is a normalized change in integral transmitted intensity. The ratio of $\mathrm{I} / \mathrm{I}_{0}$ was determined due to the transmittance characteristics of the HDN (Figure 8). Since $I / I_{0}=\left(P_{\text {out }} S_{0}\right) /\left(P_{\text {in }} S\right)$, where $P_{\text {in }}$ and $P_{\text {out }}$ are input and output radiation powers, $S_{0}$ and $S$ are the beam squares into the sample, and near the outer surface, the ratio $\mathrm{P}_{\text {out }} / \mathrm{P}_{\text {in }}$ was defined from Figure 8 and $S / S_{0}$ negligibly exceeds the unite.

Linear behaviour of the HDN transmittance under increasing optical field is not reflected in the real behaviour that was most detailed in the study by z-scan with open aperture and will 
be discussed below. However, the z-scans have shown the changes of absorption $\Delta \mathrm{T}<5 \%$ that is absolutely imperceptible in Figure 8.

It is necessary to clarify that the z-scan of low-intensity continuous radiation gives information about the total impact of all physical processes, excited by radiation in the matrix and nanoparticles, on the optical properties of the HDN.Thus, we have to divide the matrix effect from the nonlinear response caused by presence of nanoparticles.

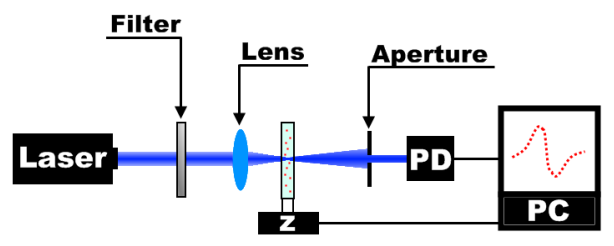

Figure 7. The experimental setup used for z-scan experiments. Setup includes: semiconductor sources of coherent continuous radiation (wavelengths of 532 and $442 \mathrm{~nm}$ with maximum power 22 and $35 \mathrm{~mW}$ and beam diameters 1,1 and $0,95 \mathrm{~mm}$, respectively); the PD-power photodetector; $75 \mathrm{~mm}$ (focus diameters $\omega_{0}=71 \mathrm{mkm}$ for the green radiation and $92 \mathrm{mkm}$ for the violet) and $50 \mathrm{~mm}\left(\omega_{0}=46 \mathrm{mkm}\right.$ for the green radiation and $90 \mathrm{mkm}$ for the violet) lens; $Z$ is $Z \mathrm{shift}$ and $\mathrm{PC}$ is a computer.
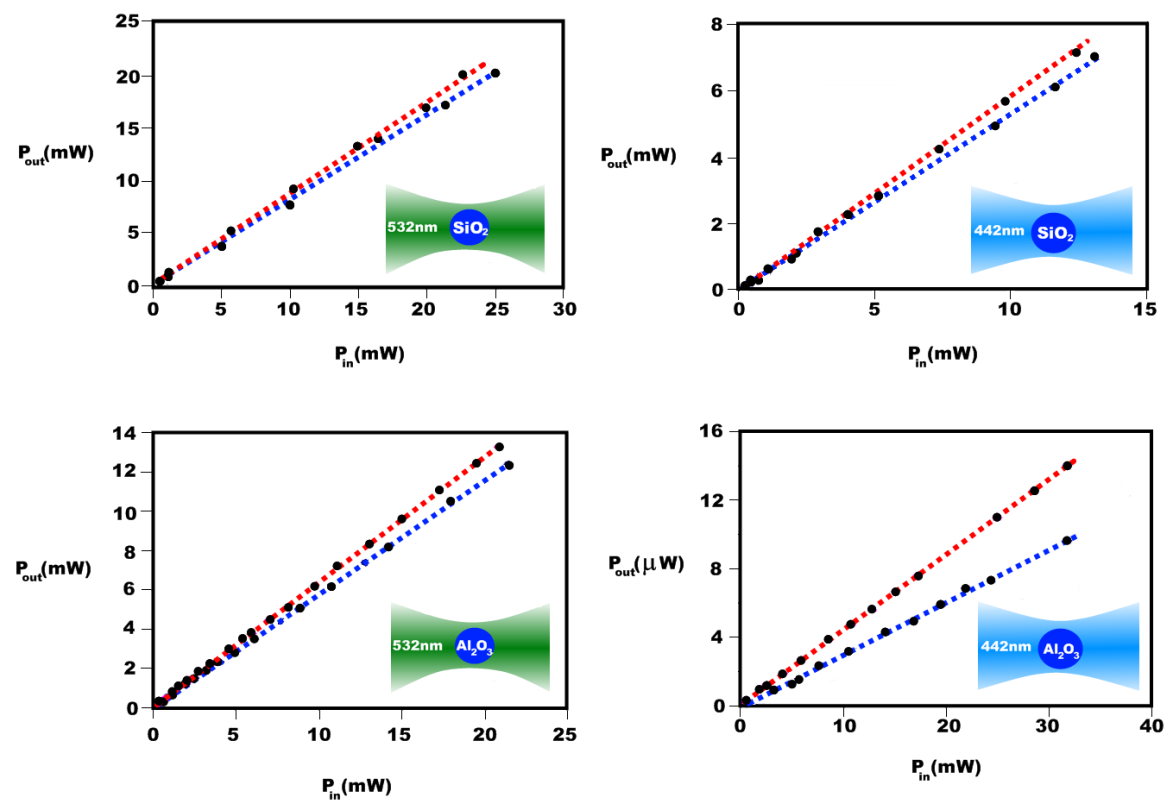

Figure 8. The integral output power $\mathrm{P}_{\text {out }}$ as a function of input power $\mathrm{P}_{\text {in }}$ of green and violet radiation propagating in the oil (red curve) and the HDN (blue curve). 
The results of measurements of the change in $\Delta \mathrm{n}$ of the $\mathrm{HDN}$ based on $\mathrm{Al}_{2} \mathrm{O}_{3}$ nanoparticles obtained by z-scan are shown in Figure 9. Considering the absorption of radiation, we used z-scans with open aperture [9].The obtained results demonstrate a linear absorption of green radiation in the samples of pure oil and the HDN (normalized transmittance is equal to 1 for all $\mathrm{z}$ ) within intensity range $(0 ; 300) \mathrm{W} / \mathrm{cm}^{2}$.However, the experiments revealed an absorption saturation of violet radiation. These experimental data have been used to adjust the normalized transmittance curves obtained with the use of the closed aperture technique, in accordance with the well-known method $[47,48]$.

The results of the z-scan with closed aperture revealed the negative thermal change in the matrix refractive index and nonlinear negative change in the HDN refractive index. Since all physical processes in the matrix and nanoparticles, forming the change of the HDN refractive index under optical field, can be considered to a first approximation as independent processes $\Delta \mathrm{n}_{\mathrm{HDN}}=\Delta \mathrm{n}_{\text {matrix }}+\Delta \mathrm{n}_{\text {nanopart }}$ so we can assume that $\Delta \mathrm{n}(\mathrm{I}, \lambda)$ for the HDN is a nonlinear function of input radiation intensity (Figure 9).

To study the effect of the matrix static permittivity on the electronic structure and optical properties of nanoparticles' array, we also investigated $\mathrm{Al}_{2} \mathrm{O}_{3}$ nanoparticles suspended in isopropyl alcohol and distilled water according to the $\mathrm{z}$-scan technique.The experiment showed a total absence of the nonlinear response in these media, being in agreement with our description in the introduction.
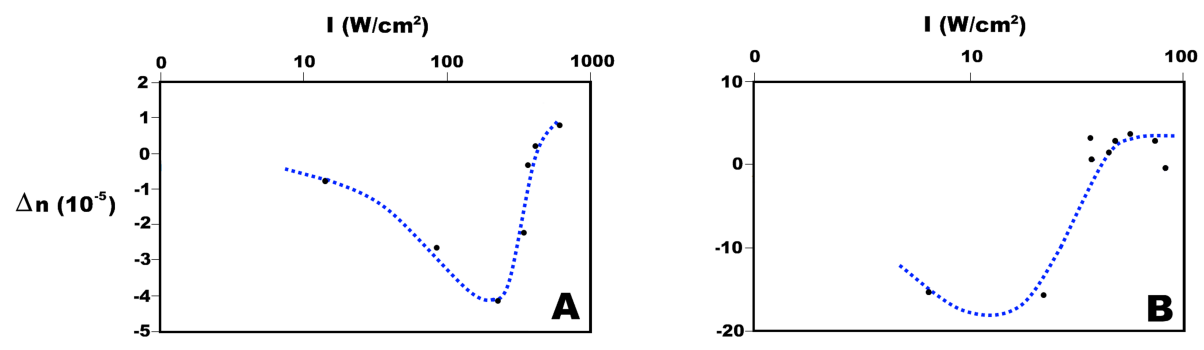

Figure 9. Change of refractive index $\Delta \mathrm{n}(\mathrm{I}, \lambda)$ of the $\mathrm{HDN}$ based on $\mathrm{Al}_{2} \mathrm{O}_{3}$ nanoparticles under green $(A)$ and violet (B) radiation. The curves were obtained by division of $\Delta n$ for the HDN by $\Delta n$ for the immersion oil.

The results of measurements of the refractive index and absorption coefficient of the HDN based on $\mathrm{SiO}_{2}$ obtained by z-scan are shown in Figures 10 and 11. Since the transformer oil characteristics are not significantly changed within intensity range $(0 ; 500) \mathrm{W} / \mathrm{cm}^{2}$, so the zscan with close and open aperture informs only about changes in the HDN optical parameters caused by nanoparticles. The obtained results demonstrate the nonlinear refraction of laser radiation (Figure 10A,C and $11 \mathrm{~A}, \mathrm{C}$ ) in the $\mathrm{HDN}$ within intensity range $(0 ; 300) \mathrm{W} / \mathrm{cm}^{2}$. In addition, the experiments revealed the nonlinear absorption of green and violet radiation in the HDN (Figure 10B,D and 11B,D) within the same intensity range. However, the HDN based on the nanoparticles of the narrow-band dielectrics $\left(\mathrm{TiO}_{2}\right.$ and $\left.\mathrm{ZnO}\right)$ did not reveal any nonlinear changes in refraction and absorption under continuous low-intensity radiation. 

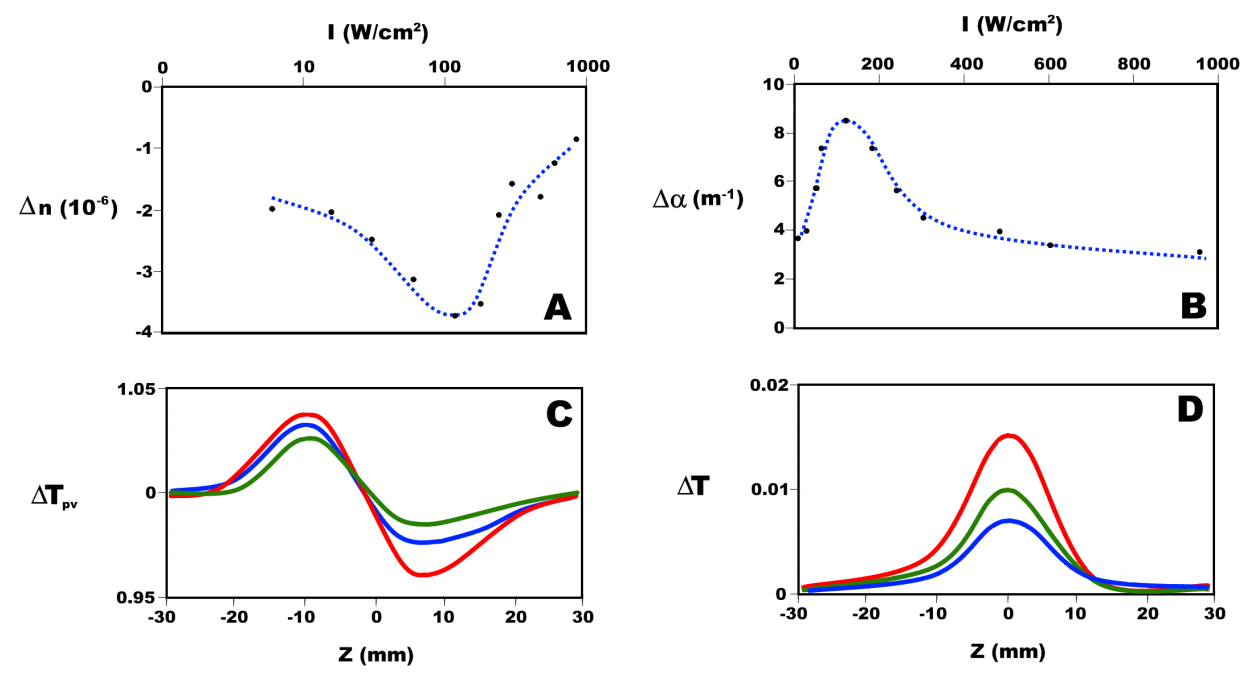

$I=30 W / \mathrm{cm}^{2}, \longrightarrow I=120 W / \mathrm{cm}^{2}$,

Figure 10. Change of refractive index $\Delta \mathrm{n}(\mathrm{A})$ and absorption coefficient $\Delta \mathrm{a}(\mathrm{B})$ of the $\mathrm{HDN}$ based on $\mathrm{SiO}_{2}$ nanoparticles under green radiation; $C$ and $D$ are the approximation of z-scan results with close and open aperture, respectively.
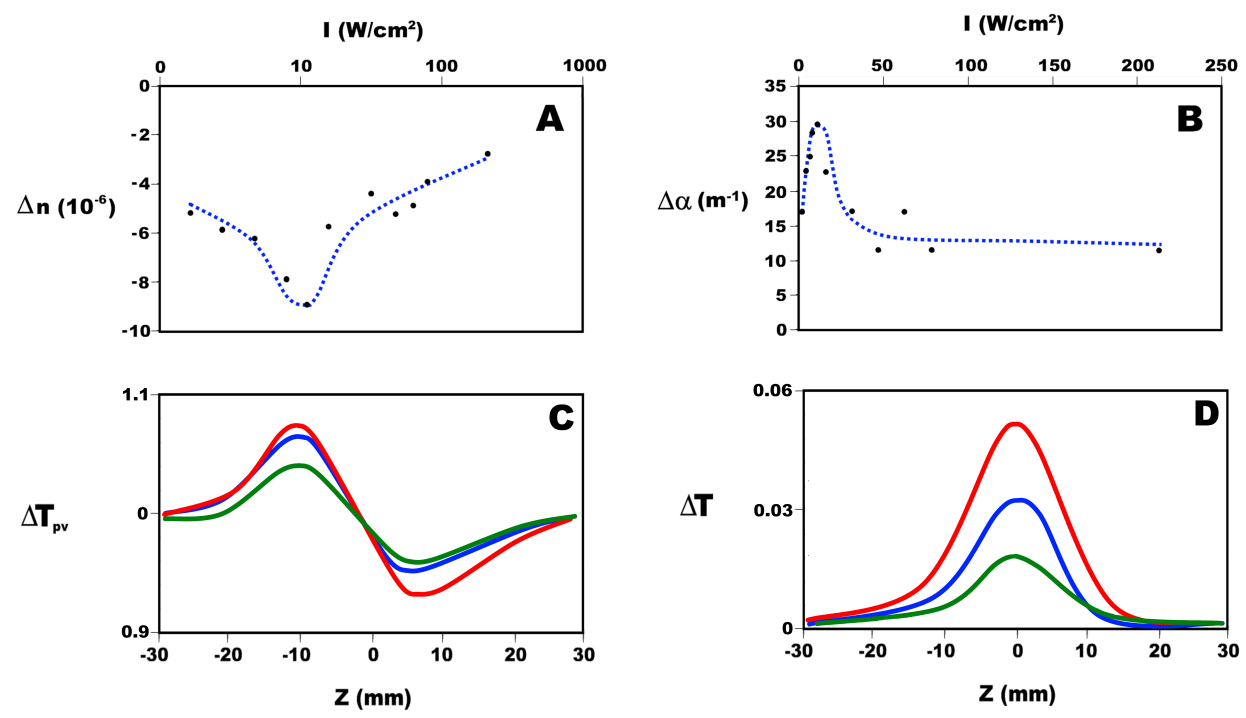

$\mathbf{I = 5} \mathbf{W} / \mathrm{cm}^{2}$,

I=11W/cm²,

I=31 $\mathbf{W} / \mathrm{cm}^{2}$

Figure 11. Change of refractive index $\Delta n(A)$ and absorption coefficient $\Delta a(B)$ of the $H_{D N}$ based on $\mathrm{SiO}_{2}$ nanoparticles under violet radiation; $C$ and $D$ are the approximation of z-scan results with close and open aperture, respectively. 


\section{The Theory of The HDN Nonlinear Transmittance}

\subsection{Preface}

The theoretical description of the physical and optical properties of heterogeneous nanocomposites containing nanoparticles is a complex problem. In fact, it seems impossible to correctly calculate the physical characteristics of an individual nanoparticle as a system consisting of a great number of particles obeying the quantum mechanics laws. Attempts to apply the well-known methods of solid state physics to describe the nanoparticles' properties run into problems, since it is not possible to disregard the effects caused by surface defects, as well as crystal lattice defects. It is known that the optical properties of a quantum mechanical system are associated with the features of the energy spectrum of charge carriers (electrons and holes).

At the present time, it is beyond all question that the optical and electric properties of nanoparticles have wide differences with that of the bulk samples due to the features of the energy spectra. These differences are caused by three effects. First, the band gap of nanoparticle charge carriers contain the allowed energies zone, herewith, the energy structure defined by the high density of surface structural defects and the irregular shape of nanoparticles. Second, the excitons and discrete energy spectra are formed below and into the conduction band due to the small nanoparticle size and size-quantization effect, respectively. In turn, the size quantization effect is caused by spatial confinement of the charge carriers' wave functions. Third, the electric dipole moments of electronic transitions in such quasi zero dimensional systems can be larger than that of the bulk sample. The formation of the above mentioned states is of threshold character, herewith, the threshold depends on the nanoparticle dimensions. Specifically, for a spherical nanoparticle (with the permittivity $\varepsilon_{2}$ ) dispersed in a medium $\left(\varepsilon_{1}\right)$, such states can be formed if the nanoparticle radius $\alpha$ is smaller than some critical radius $\alpha_{\mathrm{c}}$ :

$$
a \leq a_{c}=6|\beta|^{-1} a_{e, h}
$$

where

$$
\frac{\varepsilon_{1}-\varepsilon_{2}}{\varepsilon_{1}+\varepsilon_{2}}
$$

Here $\alpha_{\mathrm{e}, \mathrm{h}}$ is the Bohr radius of charge carriers in the nanoparticle material [49].

Some properties of the quantum states' spectrum can be clarified by studying the nanocomposites' transmittance spectra. As a rule, experimental studies are concerned with the transmittance spectra of nanoparticles' arrays embedded in a solid matrix or deposited on the transparent material surface. In this case, the electronic structure of nanoparticles is substantially influenced by the matrix material and the interaction between nanoparticles. Because of these effects, it is not possible to consider the transmittance spectra as the spectra of no 
interacting nanoparticles' arrays. Nanocomposites containing low concentrations of nanoparticles almost satisfy the condition for the lack of the above mentioned interactions, however, to study the optical properties of such composites, one cannot take into account the effects of the optical field on the distribution of the particles throughout degrees of freedom. In this case, given the low-intensity radiation, the optical field effect on the coordinates of a gravity centre of a nanoparticle can be disregarded, that cannot be said about the distribution of particles throughout the rotational degrees of freedom.

At the present time, there is no well-known theoretical approach taking into account not only the characteristic of nanoparticle dimensions, but also the orientation of nanoparticles in the external field of laser radiation, the dependences of the scattering and absorption cross sections on the propagating radiation intensity. In this context, it is necessary to develop a theoretical model of the scattering and absorption cross sections in dielectrics nanocomposites with the above mentioned features of such systems.

In this study, we suggest a semiphenomenological model of the optical transmittance of the array of noninteracting small sized $\left(\alpha<\alpha_{c}\right)$ dielectric nanoparticles embedded in the dielectric matrix. We show that the basic mechanisms of low-threshold effects of nonlinear scattering and absorption of laser radiation in the HDN are: the photo induction of electric dipole moments of nanoparticles in the external optical field; the orientation of nanoparticles along the polarization direction of this field. In addition, we will discuss the behaviour of the HDN transmittance in the central frequency vicinity of the absorption band and the dependence of the band depth on the radiation intensity.

\subsection{The Theoretical Approach}

We consider the HDN consisting of the low concentration (the number of nanoparticles $N$ per unit volume) of dielectric nanoparticles embedded in an isotropic transparent dielectric matrix with a small coefficient of viscosity and linear optical properties within the visible spectral range. In our case, the multiple scattering of radiation by nanoparticles and the nanoparticles' interaction with each other can be neglected. Let us introduce two coordinate systems with the same origin (Figure 12).

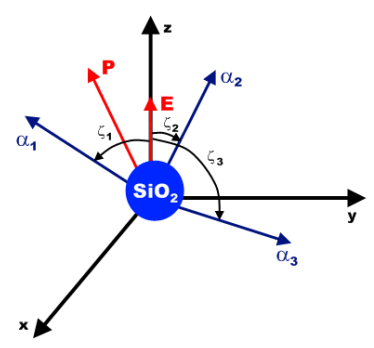

Figure 12. The coordinate system used in the theoretical study. 
One of the systems $\left\{\alpha_{1}, \alpha_{2}, \alpha_{3}\right\}$ corresponds to the coordinates coinciding with the principle axes of the particle polarization tensor with the unit vectors $\left(\mathrm{n}_{1}, \mathrm{n}_{2}, \mathrm{n}_{3}\right)$. The other system is the Cartesian laboratory coordinate system $\{x, y, z\}$ with the unit vectors $\left(\mathrm{n}_{\mathrm{x}}, \mathrm{n}_{\mathrm{y}}, \mathrm{n}_{\mathrm{z}}\right)$. We suggest that the electromagnetic wave polarized along the zaxis $E=\{0,0, E\}$ is incident on the composite. We chose the xaxis to be directed collinearly with a wave vector.

The optical transmittance of the HDN depends on the extinction coefficient, the path of the light beam in the material and the optical reflectance from the HDN boundary. For normal incidence of the light beam onto the boundary of the planar nanocomposite layer arranged normally to the xaxis, the transmittance expression can be written as [50]

$$
T(\omega, N)=\frac{\left(1-R^{2}\right)^{2} e^{\beta L}}{1-R^{2} e^{-2 \beta L}}
$$

Here, $\beta$ is the extinction coefficient, $R$ is the optical reflectance of the boundary (in experiments $\mathrm{R}$ is much smaller than unit) and $\mathrm{L}$ is the interaction length of light beam with the HDN.

In the case of single scattering approximation, the extinction coefficient can be expressed in terms of the scattering $\sigma^{s}(\omega, \mathrm{a})$ and absorption $\sigma^{\mathrm{a}}(\omega, \mathrm{a})$ cross sections of the HDN unit volume as

$$
\beta(\omega, a)=\sigma^{a}(\omega, a)+\sigma^{s}(\omega, a)+a^{m}(\omega)
$$

where $\alpha^{\mathrm{m}}(\omega)$ is the extinction coefficient of the matrix material and $a$ is the characteristic of nanoparticle dimension. For the above indicated orientation of the nanocomposite layer, the scattering and absorption cross sections in the laboratory coordinate system can be expressed in terms of the polarizability component of the HDN unit volume $\chi_{z z}(\omega, a)$ by the relations $[50,51]$

$$
\begin{aligned}
& \sigma^{a}(\omega, a)=\frac{4 \pi \omega}{c} \operatorname{Im} \chi_{z z}(\omega, a) \\
& d \sigma^{s}(\omega, a)=\frac{\omega^{4}}{c^{4}}\left|\chi_{z z}(\omega, a)\right|{ }^{2} \sin ^{2} \theta \quad d \Omega
\end{aligned}
$$

Here $\theta$ is the angle of the vector directed along the scattering direction and cis the light speed in vacuum.

We introduce the effective polarizability tensor for nanoparticle in the matrix $\alpha=\left\{\alpha_{\mathrm{ij}}\right\}$ in such a way that the components of the nanoparticle electric dipole moment Pinduced by the ex- 
ternal plane polarized monochromatic electromagnetic field $E$ with the frequency $\omega$ are determined directly in terms of the external field rather than the local field $P_{i}=\alpha_{i j} E_{j}$. In the coordinate system $\left\{\alpha_{1}, \alpha_{2}, \alpha_{3}\right\}$, the polarization vector of the nanoparticle is

$$
P=\sum_{j}^{3} a_{i j}\left(n_{j} E\right) n_{j}
$$

If the vector $\mathrm{E}$ is directed along the $\mathrm{z}$ axis, the zcomponent of the polarization vector is

$$
P_{z}=\sum_{j}^{3} a_{i j} E\left(n_{j} n_{z}\right)^{2} \sum_{j}^{3} a_{i j} E \cos ^{2} \theta_{j}
$$

Here $\theta_{\mathrm{j}}$ is the angle between vector $\mathrm{E}$ and the $\alpha_{\mathrm{j}}$ axis; this angle specifies the nanoparticle orientation in the external electromagnetic field in the laboratory coordinate system. Since the nanoparticles are randomly oriented, we assume that the polarizability tensor of the medium $\chi=\left\{\chi_{\mathrm{ij}}\right\}$ is diagonal and the polarization vector of the HDN unit volume in the laboratory coordinate system is $P_{z}=\chi_{z z} E$. Comparing this expression with (11), we obtain

$$
\chi_{z z}=N\left(a_{11} \cos ^{2} \theta_{1}+a_{22} \cos ^{2} \theta_{2}+a_{33} \cos ^{2} \theta_{3}\right)
$$

After simple transformation, taking into account that $\cos ^{2} \theta_{1}+\cos ^{2} \theta_{2}+\cos ^{2} \theta_{3}=1$, we can obtain an expression that relates the component of $\chi_{z z}$ in the laboratory coordinate system with the diagonal components of the nanoparticle polarizability tensor in the principle axes system:

$$
\chi_{z z}=N\left(a_{0}+\Delta a_{1} Q_{1}+\Delta a_{2} Q_{2}\right)
$$

where

$$
\begin{aligned}
& a_{0}=\frac{a_{11}+a_{22}+a_{33}}{3} \\
& \Delta a_{1}=a_{11}-a_{33} \\
& \Delta a_{2}=a_{22}-a_{33}
\end{aligned}
$$

The values averaged over all possible orientations

$$
\begin{aligned}
& Q_{1}=\left\langle\cos ^{2} \theta_{1}-\frac{1}{3}\right\rangle \\
& Q_{2}=\left\langle\cos ^{2} \theta_{2}-\frac{1}{3}\right\rangle
\end{aligned}
$$


are the orientation order parameters of the nanoparticles ensemble in the external field. The angle distribution function of nanoparticles and, hence, the order parameters $Q_{1}$ and $Q_{2}$, depend on the laser radiation intensity and, via the components $\alpha_{\mathrm{ij}}$ on the radiation frequency. The quantities $Q_{1}$ and $Q_{2}$ as functions of the intensity exhibit the saturation at $I>I$ irrespective of the matrix material.

The low-threshold nonlinear optical response takes place if the transmittance spectrum of the nanoparticles' array exhibits the broad absorption bands lacking in the bulk sample spectrum [31-33]. The polarizability tensor components $\alpha_{\mathrm{ij}}$ of the nanoparticle are to reach their maxima corresponding to dipole transitions of charge carriers from the state $<\mathrm{n}$ / to the state Ig> within this frequency region. In addition, it is known that the diagonal tensor components in the coordinate system of the principal axes within this frequency region can be expressed as [52]

$$
a_{j j}(\omega)=\sum_{n, g} \frac{\left|\left\langle n\left|e r_{j}\right| g\right\rangle\right|^{2}}{\square\left(\omega-\omega_{n g}+i \Gamma_{n g}\right)} \Delta \rho_{n g}
$$

The summation is performed over all allowed optical transitions of charge carriers of the nanoparticles with the frequency transition $\omega_{\text {ng }}$ from the states $\langle$ n $|$ to the states $|g\rangle$, being the component of the electric dipole moment of the transition $\mathrm{p}^{\mathrm{j}}{ }_{\mathrm{ng}}=<\mathrm{n}\left|\mathrm{er}_{\mathrm{j}}\right| \mathrm{g}>$ and the transitions width $\Gamma_{\mathrm{ng}}$. We can write the expression for only one nonzero polarizability tensor component in the laboratory coordinate system related to the individual nanoparticle using expressions (13) and (16), and introducing the definition $\Delta \omega_{\mathrm{ng}}=\omega-\omega_{\mathrm{ng}}$ :

$$
\chi\left(\omega, Q_{1}, Q_{2}\right)=N \sum_{n, g}\left[\frac{A_{n g} \Delta \omega_{n g}}{\square\left(\Delta \omega_{n g}^{2}+\Gamma_{n g}^{2}\right)}-i \frac{A_{n g} \Gamma_{n g}}{\square\left(\Delta \omega_{n g}^{2}+\Gamma_{n g}^{2}\right)}\right] \Delta \rho_{n g}
$$

The next definition is included into the expression (17)

$$
A_{n g}\left(Q_{1}, Q_{2}\right)=\frac{1}{3}\left|p_{n g}\right|^{2}+Q_{1}\left(\left|p_{1}^{n g}\right|^{2}-\left|p_{2}^{n g}\right|^{2}\right)+Q_{2}\left(\left|p_{3}^{n g}\right|^{2}-\left|p_{2}^{n g}\right|^{2}\right)
$$

The quantity $A_{n g}$ is proportional to the squared magnitude of the dipole moment of transitions from the state $<\mathrm{n} \mid$ to the state $\mid \mathrm{g}>$ provided certain optical radiation intensities, frequencies and specified parameters of the nanocomposite matrix. The population difference induced by radiation between the states $<\mathrm{n} \mid$ and $\mid \mathrm{g}>$ is a function of the incident radiation intensity. Using a two-level system approximation [52], this difference is

$$
\Delta \rho_{n g}(I)=\left(1-\frac{I / I_{S}}{\Delta \omega_{n g}^{2}+\Gamma_{n g}^{2}\left(1+I / I_{S}\right)} \Gamma_{n g}^{2}\right) \Delta \rho_{n g}^{0}
$$


where $\Delta \mathrm{Q}^{0}{ }_{\mathrm{ng}}$ is the thermal-equilibrium difference and $\mathrm{I}_{\mathrm{S}}$ is the intensity of saturation, when the $\Delta \mathrm{Q} / 2$ carriers are in the upper energy level. Separating the real and imaginary parts of the polarizability tensor component (17) and taking into account the expression (19) we introduce the definitions

$$
\begin{aligned}
& P=\int \sin ^{2} \theta d \Omega \\
& B_{n g}(\omega, T)=\frac{I / I_{S}}{\Delta \omega_{n g}^{2}+\Gamma_{n g}^{2}\left(1+I / I_{S}\right)^{\Gamma_{n g}^{2}}}
\end{aligned}
$$

we obtain the integrated scattering and absorption cross sections of the united volume of the HDN in a single scattering approximation:

$$
\begin{gathered}
\sigma_{a}(\omega, a, I)=\frac{4 \pi \omega N}{c \square} \sum_{n, g} \frac{A_{n g} \Gamma_{n g} \Delta \rho_{n g}^{0}}{\Delta \omega_{n g}^{0}+\Gamma_{n g}^{2}}\left(1-B_{n g}\right) \\
\sigma_{S}(\omega, a, I)= \\
\frac{\omega^{4} P N^{2}}{c^{4} \square^{2}} \sum_{n, g} \sum_{k, l}\left\{A_{n g} A_{k l} \frac{\left(\Delta \omega_{n g} \Delta \omega_{k l}+\Gamma_{n g} \Gamma_{k l}\right) \Delta \rho_{n g}^{0} \Delta \rho_{k l}^{0}}{\left(\Delta \omega_{n g}^{2}+\Gamma_{n g}^{2}\right)\left(\Delta \omega_{k l}^{2}+\Gamma_{k l}^{2}\right)}\left(1-B_{n g}\right)\left(1-B_{k l}\right)\right\}
\end{gathered}
$$

The dependence of the cross sections on the nanoparticle dimensions can be found by knowing the function of the relation between $\mathrm{A}_{\mathrm{ng}}$ and the nanoparticle dimension. Given $\alpha<\alpha_{\mathrm{c}}$ the dipole moment of the nanoparticle is proportional to its dimension. Therefore, as follows from expression (18), we can separate out the dependence of $A_{n g}$ on the nanoparticle dimension as $A_{n g}=S_{n g}(I) a^{2}$. Here, $S_{n g}(I)$ is a function of the radiation intensity and depends on the nanoparticle shape.

Let us estimate the ratio between the scattering and absorption cross sections. We assume that transitions occur from only one level $<\mathrm{n} \mid$ and the width of the excited level $\Gamma_{\mathrm{g}}$ has a lowdependence on $g$. Taking into account that the frequencies $\omega$ and $\omega_{\mathrm{g}}$ are of one magnitude order and the thermal equilibrium difference between the states is close to unity, and following the expressions (21) and (22), we obtain the next

$$
\frac{\sigma_{S}(\omega, a)}{\sigma_{a}(\omega, a)} \approx \frac{N P \omega^{3} a^{2}}{4 \pi c^{3} \square \Gamma} \sum_{n, g}\left\{S_{n g}(I) \quad\left(1-B_{n g}(I)\right)\right\}
$$

The quantity of $\sigma_{\mathrm{S}} / \sigma_{\mathrm{a}}$ does not exceed $\mathrm{N}^{*} 10^{-9}$ in any intensity region provided the nanoparticle dimensions $\alpha=(10 ; 100) \mathrm{nm}$ in the frequency range $\left(10^{13} ; 10^{16}\right) \mathrm{Hz}$ and $\Gamma=10^{9} \mathrm{~Hz}$. Given $\mathrm{N}^{*} 10^{-9}<1$, the scattering cross section can be omitted from the expression for the extinction coefficient. 
We can define $S_{n g}(I)=c_{n g} I$ and follow the expression (21) within radiation intensities region $\mathrm{I} /$ $\mathrm{I}_{\mathrm{S}}<<1$, we obtain the next

$$
\sigma_{a}(\omega, a, I) \approx \frac{4 \pi \omega N}{c \square} a^{2} I \sum_{n, g} c_{n g} \frac{\Gamma_{n g}}{\left(\Delta \omega_{n g}^{2}+\Gamma_{n g}^{2}\right)^{2}} \Delta \rho_{n g}^{0}
$$

The absorption cross section reaches a maximum at some intensity $\mathrm{I}=\mathrm{I}_{\mathrm{p}}$ under increasing radiation intensity, that follows from equation (21). It corresponds to the complete nanoparticle's orientation along the electric vector of the optical field and to the maximum value of $A_{n g}(I)$. This effect is responsible for a sharp enhancement of radiation absorption by the HDN unit volume. A further intensity increasing yields a noticeable increase of $B_{n g}(\omega, I)$ and decrease of the absorption cross section at a constant value of $A_{n g}(I)$. Given $I>>I_{S}$ the value of $\mathrm{B}_{\mathrm{ng}}(\omega, \mathrm{I})$ becomes approximately equal to unity resulting in an increase of the HDN transmittance. In this case, the absorption cross section can be written as

$$
\sigma_{a}(\omega, a, I) \approx \frac{4 \pi \omega N}{c \square} a^{2} \sum_{n, g} S_{n g} \frac{I_{S}}{I \quad \Gamma_{n g}} \Delta \rho_{n g}^{0}
$$

i.e., it is inverse proportional to the radiation intensity.

The broad optical absorption bands are manifested in the electronic structure of dielectric nanoparticles and absent in the corresponding bulk sample. In addition, the allowed electron energy sub-band (excitons, impurities, etc.) with the width $\Delta \omega_{1}$ lying in the band gap and adjoining the conduction band bottom, as well as the size-quantization levels (minibands) with the width $\Delta \omega_{2}$ in the conduction band, are typical for the electronic structure of HDN electrons.Taking into account the electronic structure of the nanoparticle, we substitute the summation over Ig> states with integration from $\left(\omega_{n}-\Delta \omega_{1}\right)$ to $\left(\omega_{n}+\Delta \omega_{2}\right)$ with state densities of exciton $g_{1}$ and quantum-size $g_{2}$ levels, respectively. Let us choose one of the absorption bands as an example. Changing the summation by the integration over the frequency in (21) and introducing the definitions

$$
\begin{aligned}
& \Delta \omega_{n}=\omega-\omega_{n} \\
& F(I)=\sqrt{\frac{I_{S}}{I+I_{S}}}
\end{aligned}
$$

we obtain the expression for the absorption cross section of light within the absorption band: 


$$
\begin{aligned}
\sigma_{a}=\frac{4 \pi \omega N a^{2}}{c \square} F(I) & {\left[g_{1} S_{1} \arctan \left(\frac{\Delta \omega_{1} \Gamma_{n} F(I)}{\Gamma_{n}^{2}+F^{2}(I) \Delta \omega_{n}\left(\Delta \omega_{n}+\Delta \omega_{1}\right)}\right)\right.} \\
& \left.+g_{2} S_{2} \arctan \left(\frac{\Delta \omega_{2} \Gamma_{n} F(I)}{\Gamma_{n}^{2}+F^{2}(I) \Delta \omega_{n}\left(\Delta \omega_{n}-\Delta \omega_{2}\right)}\right)\right]
\end{aligned}
$$

The quantities $S_{1}$ and $S_{2}$ are defined as the average form factors of nanoparticles $S_{n g}(I)$ for transitions to the upper and lower energy bands, respectively.

We may obtain the expression for the HDN optical transmittance in the absorption band with the central frequency $\omega_{\mathrm{n}}$ from expression (7) and (27):

$$
T(\omega, N, I) \approx \exp \left\{-L \frac{4 \pi \omega N}{c \square} D F(I)\right\}
$$

where

$$
\begin{aligned}
& D=a^{2} g_{1} S_{1} \arctan \left(\frac{\Delta \omega_{1} \Gamma_{n} F(I)}{\Gamma_{n}^{2}+F^{2}(I) \Delta \omega_{n}\left(\Delta \omega_{n}+\Delta \omega_{1}\right)}\right) \\
&+a^{2} g_{2} S_{2} \arctan \left(\frac{\Delta \omega_{2} \Gamma_{n} F(I)}{\Gamma_{n}^{2}+F^{2}(I) \Delta \omega_{n}\left(\Delta \omega_{n}-\Delta \omega_{2}\right)}\right)
\end{aligned}
$$

\subsection{The Theoretical Outputs and Discussion}

It follows from expression (28) that the optical transmittance of the HDN essentially depends on the laser radiation intensity I (Figure 13). This dependence exhibits a minimum $\mathrm{I}_{\mathrm{p}}$ corresponding to the lowest light transmittance of the HDN. As intensity is changed near $\mathrm{I}_{\mathrm{p}}$ we can see the effect of limitation of low-intensity radiation. The insert in Figure 13 is the experimental result obtained from Figure 10B. Since $\mathrm{I}_{\text {out }}=\mathrm{I}_{\text {in }} \mathrm{e}^{-\alpha \mathrm{L}}$ and $\mathrm{T}=\mathrm{I}_{\text {out }} / \mathrm{I}_{\text {in }}$ provided low reflection and absorption, we suppose $\mathrm{T}=\mathrm{e}^{-\alpha \mathrm{L}}$ and use data of $\alpha$ from Figure 10B. The theoretical and experimental results are in good agreement.

Curves from Figure 14 point out the basic features of the dependences of the HDN transmittance on the radiation wavelength. In the general case, the transmittance spectrum is asymmetric, since there is the difference between $\Delta \omega_{1}$ and $\Delta \omega_{2}$. The insert in this figure is the experimental spectrum of the $\mathrm{HDN}$ based on $\mathrm{SiO}_{2}$ nanoparticles (Figure 4B). The behaviour of the experimental curve reflects the features of the theoretical one.

The largest dipole moment is induced at the central frequency $\omega_{\mathrm{n}}$ in the absorption band. The expression for $\mathrm{D}$ at the central frequency of the absorption band $\left(\omega=\omega_{n}, \Delta \omega_{n}=0\right)$ is given by

$$
D=a^{2} g S\left\{\arctan \left(\frac{\Delta \omega_{1} F(I)}{\Gamma_{n}}\right)+\arctan \left(\frac{\Delta \omega_{2} F(I)}{\Gamma_{n}}\right)\right\}
$$


Therefore, the HDN transmittance near the central frequency can be written as

$$
T(I)=\exp \left\{-L \frac{4 \pi \omega_{n} N}{c \square} a^{2} g S\left\{\arctan \left(\frac{\Delta \omega_{1} F(I)}{\Gamma_{n}}\right)+\arctan \left(\frac{\Delta \omega_{2} F(I)}{\Gamma_{n}}\right)\right\} F(I)\right\}
$$

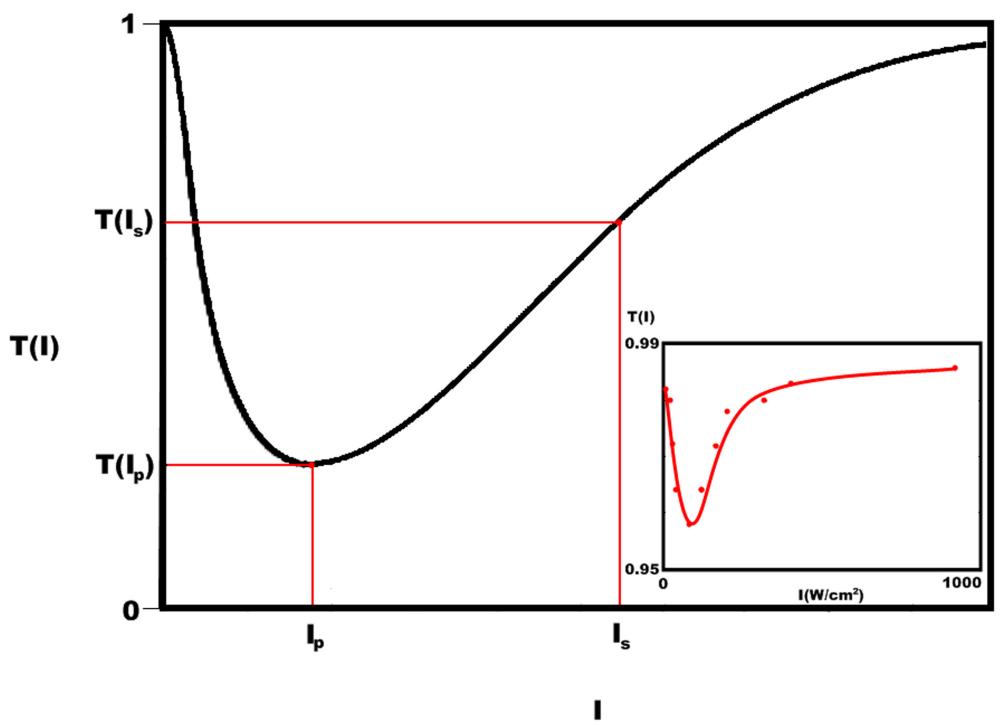

Figure 13. The theoretical dependence of the HDN transmittance on the intensity of input radiation. The insert is the experimental dependence of the HDN transmittance according with Figure 10B.

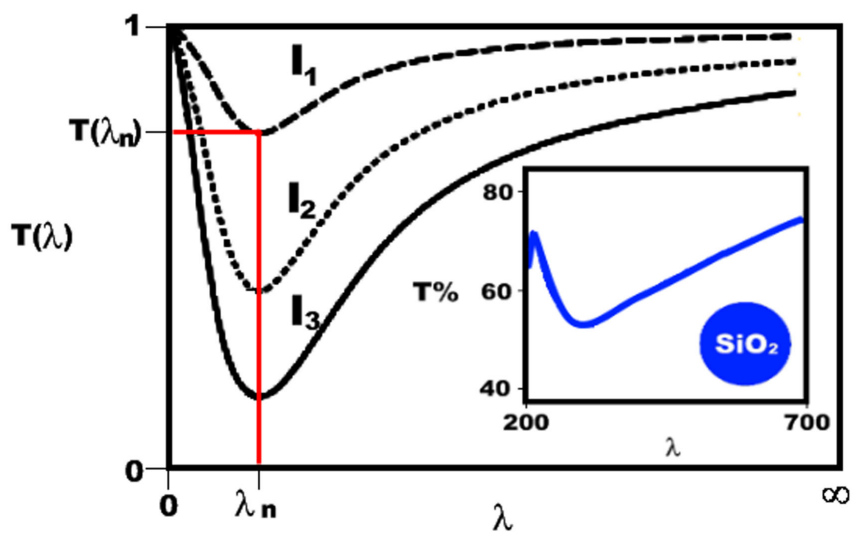

Figure 14. The theoretical dependence of the HDN transmittance on the wavelength of input radiation $\left(I_{1}>I_{2}>I_{3}\right)$. The insert is the experimental spectrum of the $\mathrm{HDN}$ based on $\mathrm{SiO}_{2}$ nanoparticles (Figure 4B). 
As one can see from expression (31), the depth of the absorption band in the transmittance spectrum depends on the radiation intensity and the nanoparticle dimension. The orientation of nanoparticles along the vector $\mathrm{E}$ requires high radiation intensities provided a solid HDN. In the case of a liquid matrix, this situation corresponds to the range of intensities $I>I_{p}$. Here we may assume that all particles are oriented along the direction of the vector $\mathrm{E}$ of the external optical field, so the order parameters are constant and $S_{n}$ are independent of the intensity. This indicates that the behaviour of the transmittance of solid and liquid matrices is similar. Therefore, the transmittance at the central frequency is

$$
T(I)=\exp \left\{-L \frac{4 \pi \omega_{n} N}{c \square \Gamma_{n}} a^{2}\left(g S_{n}\right)_{g=n}\left(\Delta \omega_{1}+\Delta \omega_{2}\right) F^{2}(I)\right\}
$$

Expression (32) exponentially approaches to unity rapidly when the radiation intensity is increased and nanoparticle dimension, the summation $\left(\Delta \omega_{1}+\Delta \omega_{2}\right)$ and multiplication of gS, become larger.

Apart from transmittance, scattering and spectral properties of the HDN, the theory can describe the behaviour of light refraction in the HDN (Figures 15,16). Using expressions (16) and (19), we can obtain the theoretical dependence of the refractive index on the intensity. Since the value of $\Delta \mathrm{n}(\mathrm{I})$ is negligible, the medium refractive index can be written as follows:

$$
n(I, \lambda) \approx n_{0}+\frac{2 \pi X_{z z}(I, \lambda)}{n_{0}}
$$

where $\mathrm{n}_{0}$ is the HDN refractive index in the absence of radiation and $\chi_{z z}$ is defined by expression (13).

Since $\chi_{\mathrm{zz}}$ is determined by $\alpha_{\mathrm{j} j}$, so we may simplify equation (16) to carry out the integration over frequency, herewith, to assume $\Gamma_{\mathrm{ng}}=\Gamma_{\mathrm{n}}$ and the state density $\mathrm{g}_{1}\left(\mathrm{~g}_{2}\right)$ and the values of $Q_{1}$ $\left(Q_{2}\right)$ are independent of the frequency $\omega$. Picking out the refraction real part from the resulting expression, we obtain $[9,45]$

$$
n(I, \omega)=n_{0}+\frac{\square}{2} \sum_{n}\left(A_{n g}\left(Q_{1}, Q_{2}\right) \Delta \rho^{0}\left(\begin{array}{c}
g_{1} \ln \frac{\left(\omega-\left(\omega_{n}-\Delta \omega_{1}\right)\right)^{2}+\Gamma_{n}^{2}\left(1+I / I_{S}\right)}{\left(\omega-\omega_{n}\right)^{2}+\Gamma_{n}^{2}\left(1+I / I_{S}\right)}+ \\
\left.+g_{2} \ln \frac{\left(\omega-\omega_{n}\right)^{2}+\Gamma_{n}^{2}\left(1+I / I_{S}\right)}{\left(\omega-\left(\omega_{n}+\Delta \omega_{2}\right)\right)^{2}+\Gamma_{n}^{2}\left(1+I / I_{S}\right.}\right)
\end{array}\right)\right.
$$

where $A_{n g}\left(Q_{1}, Q_{2}\right)$ is determined by expression (18).

Equation (34) indicates that the term $\mathrm{A}_{\mathrm{ng}}$ does not vanish in the case of propagation of unpolarized light through the medium $(\mathrm{Q}=0)$ and the nonlinear response of the dielectric nanocomposite is not equal to zero even in case of a solid matrix. 
The important conclusion from equations (18) and (34) is that the modulus of photo-induced electric dipole moments $\left|p_{n g}\right|$ mainly defines the magnitude of nonlinear optical response under continuous low-intensity radiation. In general, the orientation parametersand dipole moment modulus reach their maxima with the increase of input power, however, this increase diminishes the population difference; hence the change of $\Delta \mathrm{n}$ tends to zero. It is these two competing processes that define the nonlinear features of the HDN refractive index.

The numerical simulation of the change in the HDN refractive index was carried out using equation (34). Since the photon energy is less than nanoparticle band gap, so, the dipole transition of electrons to the exciton state is most probable $\left(g_{2}=0\right)$. In the case of the low-intensity continuous radiation and low concentration of nanoparticles, equation (34)can be rewritten as follows [9]:

$$
\Delta n(I, \omega) \approx A(I) \ln \frac{\left(\omega-\left(\omega_{n}-\Delta \omega_{1}\right)\right)^{2}+\Gamma_{n}^{2}\left(1+{ }^{I} / I_{S}\right)}{\left(\omega-\omega_{n}\right)^{2}+\Gamma_{n}^{2}\left(1+{ }^{I} / I_{S}\right)}
$$

where the factor $\mathrm{A}(\mathrm{I})$ defines the dependence of $\mathrm{A}_{\mathrm{ng}}$ on the radiation intensity as follows:

$$
A(I)=A_{0} \quad \Delta \rho^{0}\left(1-e^{-a I}\right)
$$

This dependence takes into account the magnitude of $A_{n g}$ which varies from zero to its maximum value with the increase of the external radiation intensity. The theoretical curves (Figures 15,16, solid lines) of the dependence $\Delta \mathrm{n}$ on the radiation intensity have been constructed by means of equation (35) for the $\mathrm{HDN}$ based on $\mathrm{Al}_{2} \mathrm{O}_{3}$ and $\mathrm{SiO}_{2}$ nanoparticles, irradiating by green and violet radiation. The parameters for good approximation were calculated according to the next considerations: $\Gamma$ was taken from $\mathrm{T}$ spectrum (Figure 4 ); $\mathrm{I}_{S}, \alpha$ and $\mathrm{A}_{0} \Delta \mathrm{Q}^{0}$ were calculated by means of the three-equation system (35) with known parameters I and $\Delta \mathrm{n}$ (Figures $9 \mathrm{~A}-\mathrm{B}, 10 \mathrm{~A}, 11 \mathrm{~A}$, dotted curves).

The lack of nonlinear refraction and absorption of low-intensity continuous visible laser radiation in the HDN based on the nanoparticles of narrow-band dielectrics is caused by the absence of absorption band in the used frequency range $(200 ; 700) \mathrm{nm}$. So, in order for the nonlinear optical properties of such HDN to be observable we must use high-energy pulsed radiation or change the input radiation frequency. On the one hand, if we use the pulsed radiation we may get the typical nonlinearity of the nanocomposites, caused by nonlinear behaviourof excitons near the edge of fundamental absorption (Figure 6). The high energy is required, since there is the small dipole moment of electron transition to exciton states. On the other hand, if we change the input radiation frequency it is possible to find the defect energy levels in the HDN infrared spectrum. So, the nontypical nonlinearity can take place under low-intensity infrared radiation. 

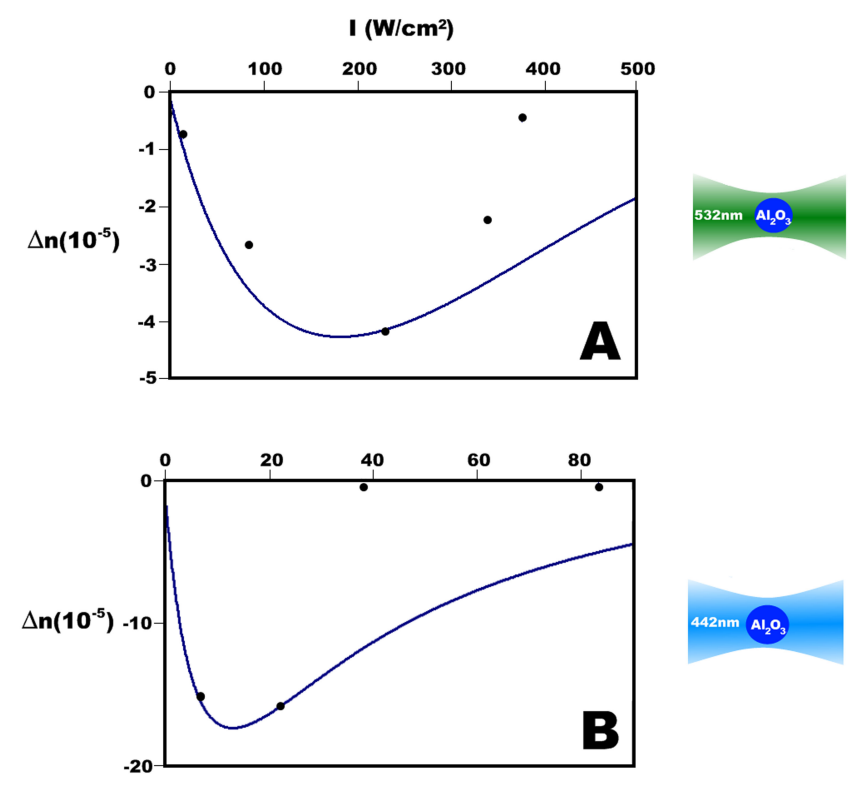

Figure 15. Theoretical curves of dependence of refraction index of green (A) and violet (B) radiation on its intensity in the $\mathrm{HDN}$ based on $\mathrm{Al}_{2} \mathrm{O}_{3}$ nanoparticles (dotted curves are the experimental results from Figure 9).
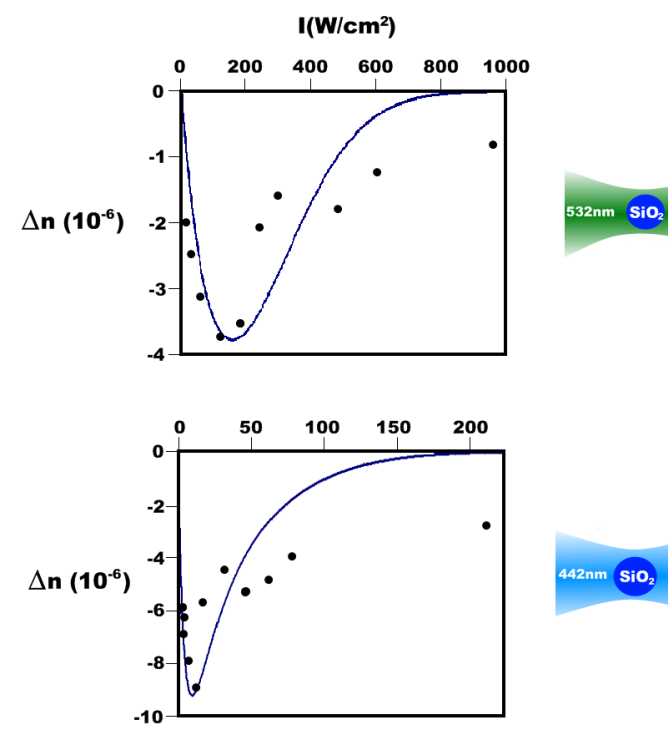

Figure 16. Theoretical curves of dependence of refraction index of green (A) and violet (B) radiation on its intensity in the $\mathrm{HDN}$ based on $\mathrm{SiO}_{2}$ nanoparticles (dotted curves are the experimental results from Figures $9 \mathrm{~A}$ and $10 \mathrm{~A}$ ). 


\section{Conclusion}

The experimental study of changes of optical characteristics of the dielectric nanostructures based on $\mathrm{Al}_{2} \mathrm{O}_{3}, \mathrm{SiO}_{2}, \mathrm{TiO}_{2}, \mathrm{ZnO}$ nanoparticles and theoretical description of these characteristics allows estimating the conditions of observing the low-threshold optical nonlinearity under low-intensity optical fields.

The ability to observe this nonlinearity is directly connected with the peculiarities of the energy spectrum of nanoparticle charge carriers. Because of the wide band gap of the bulk dielectric material, it is not possible to excite electron transitions to the conduction band by a visible light. The energy spectrum of nanoparticle electrons is of a different structure: the band gap has defect levels containing a lot of electrons due to a high density of crystal defects on the nanoparticle's surface; the small size and shape of nanoparticle leads to strong broadening of the band of high-density exciton states from the bottom of the conduction band up to defect levels. The existence of an absorption band in visible light spectrum is observed only for nanoparticles of broad-band dielectrics $\left(\mathrm{Al}_{2} \mathrm{O}_{3}, \mathrm{SiO}_{2}\right)$. The absorption band in the energy spectrum of electrons of narrow-band dielectric $\left(\mathrm{TiO}_{2}, \mathrm{ZnO}\right)$ nanoparticles is not manifested in a visible light spectrum, however, it can be manifested within the infrared region and adjoins the bottom of the conduction band.

Comparing the experimental and theoretical results we conclude that the low-threshold nonlinearity of the HDN optical parameters ( $\Delta \mathrm{n}, \Delta \alpha$ and the scattering cross section) is caused, mainly, by transitions of electrons from defect levels to exciton states and, hence, photo excitation of electric dipole moments. However, experiments have shown that the nonlinear behaviour of the HDN optical parameters takes place when the matrix permittivity $\varepsilon_{\text {stat }}$ is less than that of the nanoparticles (e.g., oil permittivity). Otherwise, the positive polarization charges, concentrated along the nanoparticle's inner surface, destroy the defect states. This explains the absence of nonlinear optical properties in the HDN based on water and alcohol matrices.

In view of the effect of giant oscillator strength, the magnitude of the photo excited dipole moment is enormous. It is the great value of the oscillator's strength for electron transition to the exciton states that is responsible for the low-threshold of the nonlinearity. As it follows from the theory, the dipole moment orientation along the external E field makes a minor contribution to the nonlinearity, therefore, this response can also be observed under pulsed and unpolarized laser radiation in solid matrices.

In addition, a qualitative agreement between experimental and theoretical results was also obtained and the proposed theory model of optical nonlinearity can be applied to explain the number of phenomena in physics of nanoscale dielectrics, e.g., proteins and blood bodies [53]. 


\section{Acknowledgements}

This work was supported by RFBR Grant No. 11-02-98514 r_vostok_a and FEB RAS Grant Nos. 12-I- OFN-05, 12-I- OFN-04, 12-I-P24-05, 12-II-UO-02-002.

\section{Author details}

Vladimir Dzyuba ${ }^{1,2^{*}}$, Yurii Kulchin ${ }^{1,2}$ and Valentin Milichko ${ }^{1,2}$

*Address all correspondence to: vdzyuba@iacp.dvo.ru

1 Institute of Automation and Control Processes of Russian Academy of Science, Vladivostok, Russia

2 Far Eastern Federal University, Vladivostok, Russia

\section{References}

[1] Alivisatos, A. P., Harris, A. L., Levinos, N. J., Steigerwald, M. L., \& Brus, L. E. (1988). Electronic states of semiconductor clusters: Homogeneous and inhomogeneous broadening of the optical spectrum. The Journal of Chemical Physics, 89(7), 4001-4011.

[2] Bawendi, M. G., Carroll, P. J., Wilson, William L., \& Brus, L. E. (1992). Luminescence properties of CdSe quantum crystallites: Resonance between interior and surface localized states. The Journal of Chemical Physics, 96(2), 946-954.

[3] Bang, Jin Ho., \& Kamat, Prashant V. (2009). Quantum Dot Sensitized Solar Cells. A Tale of Two Semiconductor Nanocrystals: CdSe and CdTe. ACS Nano, 3(6), 1467-1476.

[4] McGuire, John A., Sykora, Milan., Robel, Istva'n., Padilha, Lazaro A., Joo, Jin., Pietryga, Jeffrey. M., \& Klimov, Victor. I. (2010). Spectroscopic Signatures of Photocharging due to Hot-Carrier Transfer in Solutions of Semiconductor Nanocrystals under LowIntensity Ultraviolet Excitation. ACS Nano, 4(10), 6087-6097.

[5] Ivanov, S. A., \& Achermann, M. (2010). Spectral and Dynamic Properties of Excitons and Biexcitons in Type-II Semiconductor Nanocrystals. ACS Nano, 4(10), 5994-6000.

[6] Hashimoto, Tadanori., Yamamoto, Tsuyoshi., Kato, Tomohiro., Nasu, Hiroyuki., \& Kamiya, Kanichi. (2001). Z-scan analyses for PbO-containing glass with large optical nonlinearity. Journal of Applied Physics, 90(2), 533-537.

[7] Anderson, Mark S. (2003). Enhanced infrared absorption with dielectric nanoparticles. Applied Physics Letters, 83(14), 2964-2966. 
[8] Miheev, O. P., \& Sidorov, A. I. (2004). Optical nonlinearity of nanoparticles of widegap semiconductors and insulators in visible and near infrared spectral region. Technical Physics, 74(6), 77-82.

[9] Dzyuba, Vladimir., Milichko, Valentin., \& Kulchin, Yurii. (2011). Nontypical photoinduced optical nonlinearity of dielectric nanostructures. Journal of Nanophotonics, 5, 053528, 1-13.

[10] Yu, Baolong, Zhu, Congshan, \& Gan, Fuxi. (1997). Optical nonlinearity of Bi2O3 nanoparticles studied by Z-scan technique. Journal of Applied Physics, 82(9), 4532-4537.

[11] Major, A., Yoshino, F., Aitchison, J. S., \& Smith, P. W. E. (2004). Ultrafast nonresonant third-order optical nonlinearities in ZnSe for photonic switching at telecom wavelengths. Applied Physics Letters, 85(20), 4606-4608.

[12] He, J., Ji, W., Ma, G. H., Tang, S. H., Elim, H. I., Sun, W. X., Zhang, Z. H., \& Chin, W. S. (2004). Excitonic nonlinear absorption in CdS nanocrystals studied using Z-scan technique. Journal of Applied Physics, 95(11), 6381-6386.

[13] Larciprete, M. C., Ostuni, R., Belardini, A., Alonzo, M., Leahu, G., Fazio, E., Sibilia, C., \& Bertolotti, M. (2007). Nonlinear optical absorption of zinc-phthalocyanines in polymeric matrix. Photonics and Nanostructures - Fundamentals and Applications, 5, 73-78.

[14] Ganeev, R. A., Suzuki, M., Baba, M., Ichihara, M., \& Kuroda, H. (2008). Low- and high-order nonlinear optical properties of $\mathrm{BaTiO} 3$ and $\mathrm{SrTiO} 3$ nanoparticles. Journal of Optical Society of America B, 25(3), 325-333.

[15] Ganeev, R. A., Zakirov, A. S., Boltaev, G. S., Tugushev, R. I., Usmanov, T., Khabibullaev, P. K., Kang, T. W., \& Saidov, A. A. (2003). Structural, optical, and nonlinear optical absorption/refraction studies of the manganese nanoparticles prepared by laser ablation in ethanol. Optical Quantum Electronics, 35, 419-423.

[16] Ganeev, R. A., Ryasnyansky, A. I., Tugushev, R. I., \& Usmanov, T. (2003). Investigation of nonlinear refraction and nonlinear absorption of semiconductor nanoparticle solutions prepared by laser ablation. Journal of Optics A, 5, 409-417.

[17] Ganeev, R. A., \& Usmanov, T. (2007). Nonlinear-optical parameters of various media. IOP Quantum Electron, 37(7), 605-622.

[18] Kulchin, Yu. N., Shcherbakov, A. V., Dzyuba, V. P., \& Voznesenskiy, S. S. (2009). Interaction of collinear light beams with different wavelengths in a heterogeneous liquid-phase nanocomposite. Technical Physics Letters, 35(7), 640-642.

[19] Chen, Fang-Chung., Chu, Chih-Wei., He, Jun., Yang, Yang., \& Lin, Jen-Lien. (2004). Organic thin-film transistors with nanocomposite dielectric gate insulator. Applied Physics Letters, 85(15), 3295-3297.

[20] Schrier, Joshua., Demchenko, Denis O., Wang, Lin-Wang., \& Alivisatos, A. Paul. (2007). Optical Properties of $\mathrm{ZnO} / \mathrm{ZnS}$ and $\mathrm{ZnO} / \mathrm{ZnTe}$ Heterostructures for Photovoltaic Applications. Nano Letters, 7(8), 2377-2382. 
[21] Dutta, Kousik., \& De, S. K. (2007). Electrical conductivity and dielectric properties of $\mathrm{SiO} 2$ nanoparticles dispersed in conducting polymer matrix. Journal of Nanoparticle Research, 9, 631-638.

[22] Chu, Daobao, Yuan, Ximei, Qin, Guoxu, Xu, Mai, Zheng, Peng, Lu, Jia, \& Zha, Longwu. (2008). Efficient carbon-doped nanostructured TiO2 (anatase) film for photoelectrochemical solar cells. Journal of Nanoparticle Research, 10, 357-363.

[23] Liu, Li., Wang, Ning., Cao, Xia., \& Guo, Lin. (2010). Direct Electrochemistry of Cytochrome c at a Hierarchically Nanostructured TiO2 Quantum Electrode. Nano Research, 3-369.

[24] Hensel, Jennifer., Wang, Gongming., Li, Yat., \& Zhang, Jin. Z. (2010). Synergistic Effect of CdSe Quantum Dot Sensitization and Nitrogen Doping of TiO2 Nanostructures for Photoelectrochemical Solar Hydrogen Generation. Nano Letters, 10, 478-483.

[25] Pellegrini, Giovanni., Mattei, Giovanni., \& Mazzoldi, Paolo. (2009). Light Extraction with Dielectric Nanoantenna Arrays. ACS Nano, 3(9), 2715-2721.

[26] Redel, Engelbert., Mirtchev, Peter., Huai, Chen., Petrov, Srebri., \& Ozin, Geoffrey. A. (2011). Nanoparticle Films and Photonic Crystal Multilayers from Colloidally Stable, Size-Controllable Zinc and Iron Oxide Nanoparticles. ACS Nano, 5(4), 2861-2869.

[27] Chen, F. C., Chuang, C. S., Lin, Y. S., Kung, L. J., Chen, T. H., \& Shien, D. H. P. (2006). Low-voltage organic thin-film transistors with polymeric nanocomposite dielectrics. Organic Electronics, 7, 435-439.

[28] Park, Young-Shin., Cook, Andrew K., \& Wang, Hailin. (2006). Cavity QED with Diamond Nanocrystals and Silica Microspheres. Nano Letters, 6(9), 2075-2079.

[29] Han, Jiaguang., Woo, Boon Kuan., Chen, Wei., Sang, Mei., Lu, Xinchao., \& Zhang, Weili. (2008). Terahertz dielectric properties of $\mathrm{MgO}$ nanocristals. The Journal of Physical Chemistry C, 112, 17512-17516.

[30] Balasubramanian, Balamurugan., Kraemer, Kristin L., Reding, Nicholas A., Skomski, Ralph., Ducharme, Stephen., \& Sellmyer, David J. (2010). Synthesis of Monodisperse TiO2-Paraffin Core-Shell Nanoparticles for Improved Dielectric Properties. ACS Nano, 4(4), 1893-1900.

[31] Landes, C., Braun, M., Burda, C., \& El -Sayed, M. A. (2001). Observation of Large Changes in the Band Gap Absorption Energy of Small CdSe Nanoparticles Induced by the Adsorption of a Strong Hole Acceptor. Nano Letters, 1(11), 667-670.

[32] Kulchin, Yu. N., Shcherbakov, A. V., Dzyuba, V. P., Voznesenskii, S. S., \& Mikaelyan, G. T. (2008). Nonlinear-optical properties of heterogeneous liquid nanophase composites based on high-energy-gap A12O3 nanoparticles. IOP Quantum Electronics, 38(2), 154-158. 
[33] Ho, Ching-Hwa., Chan, Ching-Hsiang., Tien, Li-Chia., \& Huang, Ying-Sheng. (2011). Direct Optical Observation of Band-Edge Excitons, Band Gap, and Fermi Level in Degenerate Semiconducting Oxide Nanowires In2O3. Journal of Physical Chemistry C, 115, 25088-25096.

[34] Mu, R., Tung, Y. S., Ueda, A., \& Henderson, D. O. (1996). Chemical and Size Characterization of Layered Lead Iodide Quantum Dots via Optical Spectroscopy and Atomic Force Microscopy. Journal of Physical Chemistry, 100, 19927-19932.

[35] Li, Jingbo., \& Wang, Lin-Wang. (2003). Shape Effects on Electronic States of Nanocrystals. Nano Letters, 3(10), 1357-1363.

[36] Al-Hilli, S. M., \& Willander, M. (2006). Optical properties of zinc oxide nano-particles embedded in dielectric medium for UV region: Numerical simulation. Journal of Nanoparticle Research, 8, 79-97.

[37] Stroyuk, Oleksandr. L., Dzhagan, Volodymyr. M., Shvalagin, Vitaliy. V., \& Kuchmiy, Stepan Ya. (2010). Size-Dependent Optical Properties of Colloidal ZnO Nanoparticles Charged by Photoexcitation. The Journal of Physical Chemistry C, 114, 220-225.

[38] Azpiroz, Jon M., Mosconi, Edoardo., \& De Angelis, Filippo. (2011). Modeling ZnS and ZnO Nanostructures: Structural, Electronic, and Optical Properties. The Journal of Physical Chemistry C, 115, 25219-25226.

[39] Pokutnyi, S. I. (2006). Optical absorption and scattering at one-particle states of charge carriers in semiconductor quantum dots. Semiconductor, 40(2), 217-223.

[40] Pokutnyi, S. I. (2007). Excition states in semiconductor quantum dots in the modified effective mass approximation. Semiconductor, 41(11), 1323-1328.

[41] Sinha, Sucharita., Ray, Alok., \& Dasgupta, K. (2000). Solvent dependent nonlinear refraction in organic dye solution. Journal of Applied Physics, 87(7), 3222-3226.

[42] Mosconi, Edoardo., Selloni, Annabella., \& De Angelis, Filippo. (2012). Solvent Effects on the Adsorption Geometry and Electronic Structure of Dye-Sensitized TiO2: A First-Principles Investigation. Journal of Physical Chemistry C, 116, 5932-5940.

[43] Dzyuba, V. P., Krasnok, A. E., Kulchin, Yu. N., \& Dzyuba, I. V. (2011). A model of nonlinear optical transmittance for insulator nanocomposites. Semiconductor, 45(3), 295-301.

[44] Dzyuba, V. P., Krasnok, A. E., \& Kulchin, Yu. N. (2010). Nonlinear refractive index of dielectric nanocomposites in weak optical fields. Technical Physics Letters, 36(11), 973-977.

[45] Kulchin, Yu. N., Dzyuba, V. P., \& Voznesenskiy, S. S. (2011). Threshold optical nonlinearity of dielectric nanocomposite. In: Boreddy Reddy (ed.), Advances in Diverse Industrial Applications of Nanocomposites, Rijeka, InTech, 261-288.

[46] Wakaki, M., Kudo, K., \& Shibuya, T. (2007). Physical Properties and Data of Optical Materials. CRC Press Taylor and Francis Group. New York. 10.1201/9781420015508 
[47] Said, A. A., Sheik-Bahae, M., Hagan, D. J., Wei, T. H., Wang, J., Young, J., \& Van Stryland, E. W. (1991). Determination of bound-electronic and free-carrier nonlinearities in ZnSe, GaAs, CdTe and ZnTe. Journal of Optical Society of America B, 9(3), 405-414.

[48] Van Stryland, E. W., \& Sheik-Bahae, M. (1998). Z-scan measurement of optical nonlinearities. In: Kuzyk M. G., DirkC. W. (eds), Characterization Techniques and Tabulation for Organic Nonlinear Materials, Marcel Dekker Inc., New York, 655-692.

[49] Pokitnyi, S. I., \& Efremov, N. A. (1991). Theory of Macroscopic Local Single-Particle Charge States in Quasi-Zero-Dimensional Structures Surface Local States. Phys. Status Solidi B, 165, 109-118.

[50] Bohren, C., \& Huffman, D. (1983). Absorbtion and Scattering of Light by Small Particles, Wiley, New York.

[51] Landau, L. D., \& Lifshitz, E. M. (1984). Course of Theoretical Physics, Volume 8: Electrodynamics of Continuous Media. Pergamon, New York.

[52] Shen, Y. R. (1984). The Principles of Nonlinear Optic. Wiley, Hoboken, NJ.

[53] Kulchin, Yu. N., Dzyuba, V. P., \& Milichko, V. A. (2010). Optical Nonlinearity of a Biological Liquid Nanocomposite. Pacific Science Review, 12(1), 4-7. 